\title{
The mitochondrial genomes of sponges provide evidence for multiple invasions by Repetitive Hairpin-forming Elements (RHE) Dirk Erpenbeck* ${ }^{* 1}$, Oliver Voigt ${ }^{1}$, Gert Wörheide ${ }^{1}$ and Dennis V Lavrov ${ }^{2}$
}

Address: ${ }^{1}$ Department of Earth- and Environmental Sciences, Palaeontology \& Geobiology and GeoBioCenter LMU, Ludwig-Maximilians Universität München, Richard-Wagner-Str. 10, 80333 München, Germany and 2Department of Ecology, Evolution, and Organismal Biology, Iowa State University, 343A Bessey Hall, Ames, IA 50011, USA

Email: Dirk Erpenbeck* - erpenbeck@lmu.de; Oliver Voigt - oliver.voigt@lmu.de; Gert Wörheide - woerheide@lmu.de; Dennis V Lavrov - dlavrov@iastate.edu

* Corresponding author

Published: 9 December 2009

BMC Genomics 2009, 10:591
Received: 12 February 2009

Accepted: 9 December 2009

This article is available from: http://www.biomedcentral.com/I47I-2/64/I0/59I

(c) 2009 Erpenbeck et al; licensee BioMed Central Ltd.

This is an Open Access article distributed under the terms of the Creative Commons Attribution License (http://creativecommons.org/licenses/by/2.0), which permits unrestricted use, distribution, and reproduction in any medium, provided the original work is properly cited.

\begin{abstract}
Background: The mitochondrial $(\mathrm{mt})$ genomes of sponges possess a variety of features, which appear to be intermediate between those of Eumetazoa and non-metazoan opisthokonts. Among these features is the presence of long intergenic regions, which are common in other eukaryotes, but generally absent in Eumetazoa. Here we analyse poriferan mitochondrial intergenic regions, paying particular attention to repetitive sequences within them. In this context we introduce the mitochondrial genome of Ircinia strobilina (Lamarck, 1816; Demospongiae: Dictyoceratida) and compare it with mtDNA of other sponges.

Results: Mt genomes of dictyoceratid sponges are identical in gene order and content but display major differences in size and organization of intergenic regions. An even higher degree of diversity in the structure of intergenic regions was found among different orders of demosponges. One interesting observation made from such comparisons was of what appears to be recurrent invasions of sponge mitochondrial genomes by repetitive hairpin-forming elements, which cause large genome size differences even among closely related taxa. These repetitive hairpin-forming elements are structurally and compositionally divergent and display a scattered distribution throughout various groups of demosponges.

Conclusion: Large intergenic regions of poriferan $\mathrm{mt}$ genomes are targets for insertions of repetitive hairpin- forming elements, similar to the ones found in non-metazoan opisthokonts. Such elements were likely present in some lineages early in animal mitochondrial genome evolution but were subsequently lost during the reduction of intergenic regions, which occurred in the Eumetazoa lineage after the split of Porifera. Porifera acquired their elements in several independent events. Patterns of their intra-genomic dispersal can be seen in the $\mathrm{mt}$ genome of Vaceletia sp.
\end{abstract}

\section{Background}

Organellar genomes display a tendency of size reduction (deletional bias) [1]. This tendency manifests itself in the loss of mitochondrial $(\mathrm{mt})$ protein genes or their relocation to the nucleus, and in the loss of intergenic non-coding sequences. For example, comparison between animal 
$\mathrm{mt}$ genomes and that of the choanoflagellate Monosiga brevicollis revealed that a major reduction of mtDNA has taken place in the animal lineage, which involved the translocation of mitochondrial genes into the nucleus and dramatic size reduction of intergenic regions (IGR) [2]. Indeed, the IGRs account for almost $50 \%$ of the $76 \mathrm{~kb} \mathrm{mt}$ genome of M. brevicollis [3], while the poriferan genomes examined so far from Demospongiae, Homoscleromorpha and Hexactinellida (no mt genome is yet available for class Calcarea) possess less than 24\% IGRs (in Axinella corrugata [4]). Similarly, the number of genes is reduced from 55 in M. brevicollis [3] to $40-18$ in demosponges [5]. This diminution of mt DNA culminates in bilaterian animals, where IGRs are frequently absent (with adjacent genes often overlapping each other) and occasionally genes being lost. As a result, the majority of non-coding nucleotides in bilaterian $\mathrm{mt}$ genomes is located in a single control region, which contains important elements for the replication of mtDNA (see [6] for an overview). Although such control region has not unambiguously been identified in non-bilaterian Metazoa, some characteristic features like repetitive sequences [7], conserved sequence blocks and potential secondary structures for the initiation of replication [8] have been found in the $\mathrm{mt}$ genome of Acropora tenuis (Cnidaria [9]). In Porifera, non-coding regions with repetitive features are speculative control regions, but similarity to their eumetazoan counterparts and conservation among different species are low $[10,11]$.

The structure and biological function of $\mathrm{mt}$ genes is wellstudied owing to their pivotal role in respiration and oxidative phosphorylation and several of these genes are frequently used as phylogenetic markers. By contrast, elements involved in the replication and expression of mtDNA have been investigated only in a few species [8]. However, it has been observed that mtDNA outside of bilaterian animals usually contains multiple IGRs of similar length and these genomes often harbour numerous repetitive sequences [12]. Such repeats occur mostly in intergenic regions but were also found inside protein coding or ribosomal RNA genes (e.g. [13-15]). These mt repetitive sequences can comprise all classes of their nuclear counterparts, which include direct-, dispersed-, inverse-, tandem- and satellite-like repeats (see [12] for an overview). Frequently, repetitive mtDNA elements have a potential to form a particular secondary structure with stems and loops. Conserved and potentially mobile palindromic repeats are well known from non-metazoan mtDNA [16]. Most abundant are single hairpin-forming motifs (e.g. [17]), but double hairpin elements were also found $[12,18]$. In the following we will refer to such elements as Repetitive Hairpin-forming Elements (RHE).

Despite their regular occurrence in fungi, plants, and other eukaryotes, no adaptive function of RHEs is known, although their potential roles as control elements in
mRNA processing and translation have been discussed. For example, inverted repeat sequence elements are often found in the 3' - untranslated regions of mRNAs and have been suggested as candidate structures for RNAse access [19]. Alternatively, inverted repeats forming stem-loop structures at 3' termini of mRNAs have also been found to be stabilizing signals in both bacteria and chloroplasts [20-23]. Besides their involvement in RNA processing, repeat structures and, in particular, (double-) hairpin elements could facilitate recombination and lead to $\mathrm{mt}$ genome reorganization. This function would be analogous to G-C rich clusters in the $\mathrm{mt}$ genome of $S$. cerevisiae [12], which can be folded into several different motifs of stem-and-loop structures with high similarity [24] and are regarded as preferential recombination sites [25-28]. Finally, mt repetitive elements, like their cytoplasmatic counterparts, could have simply evolved out of transposable elements or by errors during mt DNA replication [12].

These insights from non-metazoan opisthokonts indicate that mt IGRs may play an important role in the evolution of the metazoan $\mathrm{mt}$ genome. To our knowledge there is no information about the presence of RHE in choanoflagellates, Ichtyosporea and Placozoa. In Bilateria, the impact of RHE was reduced concurrently with the condensation of the $\mathrm{mt}$ genome to the highly compact circular $\sim 16 \mathrm{~kb}$ DNA molecule, as present today in most animal phyla. To investigate the potential impact of RHE on early metazoan $\mathrm{mt}$ DNA evolution, we studied the IGRs in all available $\mathrm{mt}$ genomes from sponges, which form the basal divergence with Eumetazoa within the animal lineage [29]. In this paper, we will initially focus on the mt genomes of keratose demosponges (Keratosa), which, together with the Myxospongiae, form the sister group to all other extant demosponge lineages $[5,30]$ and then extend our analysis to other groups of demosponges and sponges in general.

The keratose sponge order Dictyoceratida encompasses sponges with a high morphological diversity. Most genera, such as Hippospongia or Ircinia possess a purely organic skeleton of spongin fibers. Recent molecular data, however, demonstrated that Vaceletia, a sponge with a 'sphinctozoan' -type skeleton, i.e. with a hypercalcified (so-called "coralline") mineral skeleton of aragonite with trabecular inner structure, likewise belongs to the Dictyoceratida $[5,31]$, a taxon that normally is devoid of biomineral-production. In this context we present the mt genome of an additional non-coralline dictyoceratid sponge, Ircinia strobilina, which allows us to get a better insight on intra-ordinal variation of sponge mtDNA.

\section{Results \\ Organization of dictyoceratid demosponge mtDNA}

The mt genomes of dictyoceratid demosponges Ircinia strobilina, Hippospongia lachne and Vaceletia sp. comprise 
$16,414,16,755$, and 20,658 bp, respectively (Figure 1). They code for the standard 14 demosponge protein genes (which include atp9), small and large subunit ribosomal RNA ( $r n s$ and $r n l$ ) but only two tRNAs (trnW(uca) and $\operatorname{trnM} f(\mathrm{cau}))$. The latter represent the minimally required suite of mt tRNA genes if all other tRNAs are imported from the cytoplasm: $\operatorname{trn} W(\mathrm{uca})$ is needed to accommodate for sponge (and mold and cnidarian) deviation from the universal mitochondrial code [32] while a specialized $\operatorname{trnMf(cau)}$ is necessary for the initiation of translation (see also [33]). This reduced suite of tRNA genes is identical to that found in most Cnidaria [34]. All three mt genomes display an identical gene order, including the position of tRNAs. The selection pressures on the coding genes is also very similar in these genomes as the ratio of synonymous/nonsynonymous substitutions per site is estimated as: Vaceletia sp. - I. strobilina: (0.30/0.03); Vace- letia sp. - H. lachne: (0.27/0.03); I. strobilina - H. lachne: $(0.17 / 0.02)$.

\section{Structure of intergenic regions in dictyoceratid demosponges}

Dictyoceratid intergenic regions show large length differences, resulting in mtDNA size variation of approximately $20 \%$ among the analyzed species. Vaceletia sp. possesses IGRs totalling $4,520 \mathrm{bp}$, compared to $1,566 \mathrm{bp}$ and 871 bp in H. lachne and I. strobilina, respectively. IGRs in the latter two species display a high degree of similarity (Figure 1 grey vertical bars), which is highest at IGR termini and decreases towards their centres. In Vaceletia the IGRs connecting $r n l$ - atp9 (700 bp), and trnM(cau) - nad2 (657 bp) are particularly long. There is no evidence for additional ORFs in any of these regions.

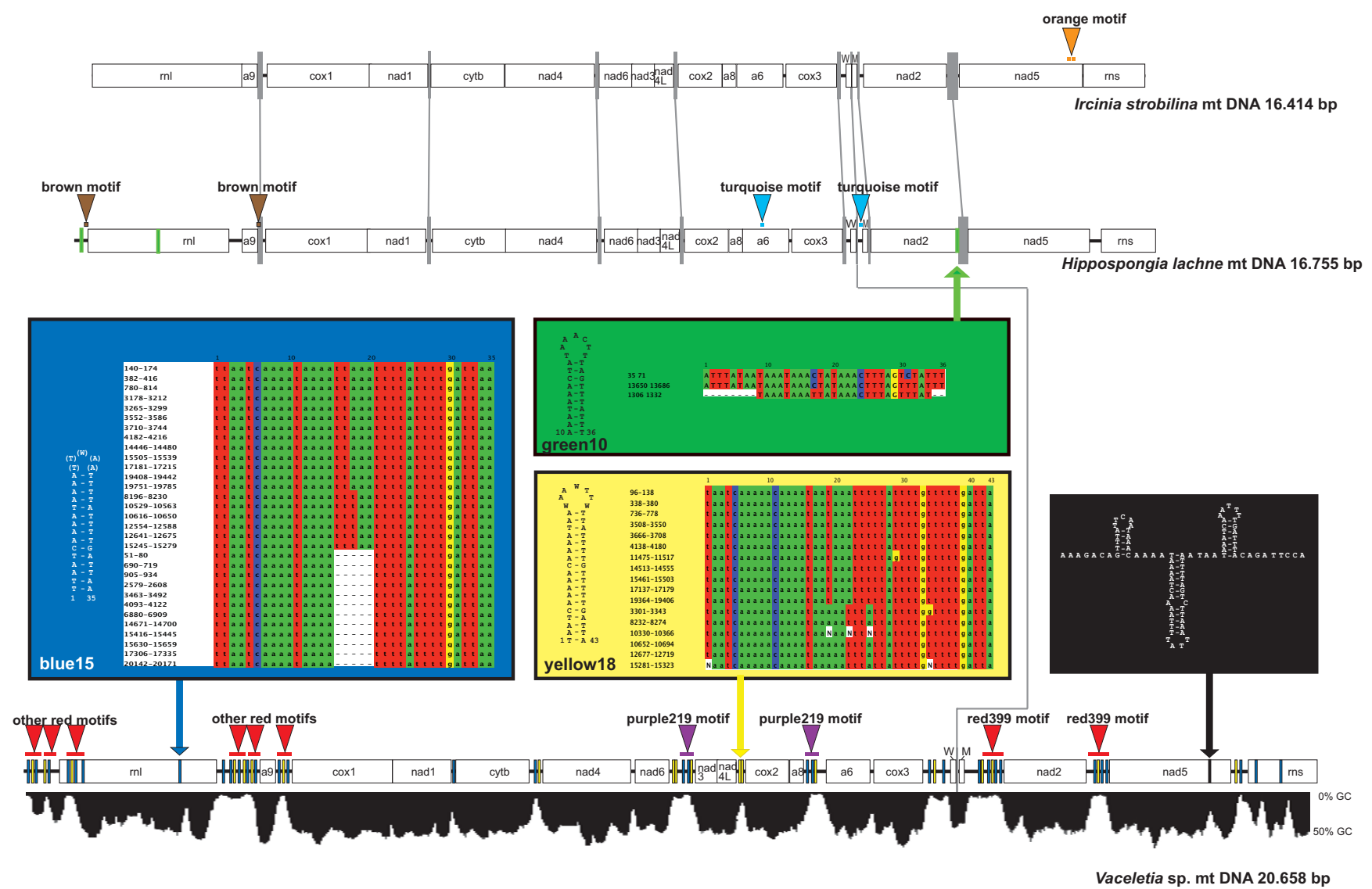

Figure I

Dictyoceratida mtDNA. Schematic and linearized view of the three dictyoceratid mt genomes. The grey vertical bars indicate IGRs of high similarity between the taxa as connected by grey lines. Coloured horizontal bars (also highlighted with triangles and motif names) indicate repetitive regions inside the genomes. Coloured vertical bars (also highlighted with triangles and motif names) inside the genomes display putative RHEs. Their corresponding secondary structure and alignment is provided inside the boxes of the same colour. The GC-content of the Vaceletia mt genome is indicated by the black field below the structure. The numbers in the sequence names refer to their position in the $\mathrm{mt}$ genome. The numbers at the structures refer to their position in the alignments. 


\section{Repetitive elements in the IGR of dictyoceratid demosponges}

Several long duplications exist within the mt IGR of Vaceletia. Two IGR stretches of 339 bp between nad5 - nad2 and $\operatorname{trnMf(cau)~-~nad2~are~identical~(Figure~1,~red~horizon-~}$ tal bars "red 339 motif"). Parts of this motif are also present in other IGRs: rnl-atp9 (108 and 219 bp), atp9-cox1 (222 bp), rns-rnl (214 and 88 bp) and even within rnl (236 bp) (Figure 1, red horizontal bars "other red motifs"). Another duplicated region of significant length (219 bp) is located in the IGRs connecting nad6 - nad3 and atp8 - atp6 (Figure 1: purple horizontal bars "purple219 motif").

These long repetitive regions are more than 90\% AT, which is much higher than for the other regions of the $\mathrm{mt}$ genome (Figure 1, black curve under the Vaceletia $\mathrm{mt}$ genome). A closer analysis of the AT-rich repetitive regions reveals the presence of repetitive small, subunits, which form perfect hairpin structures. Two different RHE types have been detected: the first type consists of two uninterrupted complementary regions of 15 bp each, occasionally separated by 5 bases, which will in the following be referred to as "blue15" (Figure 1). We have found 32 complete, i.e. hairpin-forming copies of this type. The second type consists of two complementary regions of $18 \mathrm{bp}$ with a $7 \mathrm{bp}$ terminal loop (Figure 1 ). The Vaceletia mt genome contains 17 complete copies of this type, which will in the following be referred to as "yellow18". Besides their length, the blue15 and yellow18 complementary regions differ from each other by few complementary substitutions. In addition, several incomplete blue15 and yellow18 RHE (i.e. without complementary region in close proximity) have been detected (not shown). The blue15 and yellow18 RHE often occur in tandem resulting in double hairpin and, in some cases, multiple hairpin structure. Both hairpin motifs also occur in rnl, while the blue15 type is also present in rns.

Compared to its homologues in dictyoceratid species Hippospongia lachne and Ircinia strobilina, nad5 of Vaceletia sp. possesses a 90 bp AT-rich insert (Figure 1: black vertical bar) with a potential to form a triple hairpin structure (Figure 1, black box). Similar insertions have been found in nad2 and nad5 of Axinella corrugata (see below). Considering the fact that they do not cause frameshifts it is likely that these elements are not spliced out of the transcripts. Also, insertions in nad5 are not unusual in Order Dictyoceratida - Ircinia strobilina possesses a stretch of 16 almost identically duplicated amino acids at the 3 ' end (YVT(VW/GS)GIEYAEVPEYL), Figure 1, orange elements "orange motif"). In the Ircinia strobilina mt genome this is the only repetitive feature.

In contrast to our findings in Vaceletia, the mt genome of Hippospongia lachne possesses only three repetitive motifs.
The first forms a RHE type with a 10 bp helix and a 7 bp loop in three copies (Figure 1: "green10"), of which two occur in IGRs and one in $r n l$ (Figure 1: green vertical bar). In addition, a $31 \mathrm{bp}$ long fragment of atp 6 has been copied into the IGR between the tRNA genes (Figure 1, "turquoise motifs") and a 42 bp fragment is identical in the intergenic regions 5' of $r n l$ and $\operatorname{cox} 1$ (Figure 1, "brown motif"). Both of the latter elements do not form hairpins.

\section{Repetitive and other unusual elements in mtDNA of other demosponges \\ Keratosa}

Dendroceratida is sister order to Dictyoceratida - both form the taxon Keratosa. MtDNA of the dendroceratid Igernella notabilis possesses IGRs of 3,586 nucleotides. The most remarkable feature of these IGRs is the presence of a highly AT-rich RHE in 14 complete, i.e. double- or triple hairpin-forming copies (Figure 2A), besides several potentially incomplete copies. Complete RHE copies are found in all intergenic regions with the exception of cox1-nad1, cox2-atp8, cob-nad6, nad3-nad4l, nad4-trnM, nad2trnW(uca) and rns-atp9, but twice between trnW(uca) and nad5, and one such element is inserted into $\mathrm{rnl}$.

\section{Myxospongiae}

Sponges of the orders Halisarcida, Chondrosida and Verongida are combined into the Myxospongiae [30] and form a sister group to Keratosa in molecular phylogenies [5,30]. The IGR of Halisarca dujardini (Halisarcida) contains 1,328-noncoding base pairs with only one potential RHE type, 17 bp long and GC-rich (Figure 2B). The RHEs are located in the IGRs connecting $\operatorname{cox} 3-\operatorname{trn} Q(u u g)$, atp9 $\operatorname{trnS}(\mathrm{gcu})$ and, furthermore, inside a variable region of nad2. Chondrilla nucula (Chondrillidae) does not possess any remarkable repetitive feature in its 1,377 bp intergenic regions. Aplysina fulva (Verongida) has 1,478 intergenic nucleotides, with just a single RHE motif of two complementary 10 bp strands. It occurs in two copies in the IGRs connecting $\operatorname{trnH}(\mathrm{gug})$ - nad4 and atp6 - trnR(ucu) (Figure 2C).

\section{Marine Haplosclerida}

Mt genomes of marine haplosclerids Callyspongia plicifera (1,100 bp), Xestospongia muta (938 bp) Amphimedon compressa (887 bp) and Amphimedon queenslandica (2,413 bp) lack RHEs. However, Amphimedon queenslandica $(2,413$ bp) mtDNA contains a $6 \times$ tandemly repeated 12 bp motif between the two rRNA genes, a feature that remains unique among Porifera [10].

\section{The "G4" clade}

According to molecular markers [5,30], the remaining demosponge lineages belong to the clade "G4" yet to be named and resolved. The mt genome of the freshwater sponge Ephydatia muelleri (Haplosclerida, Spongillina) 

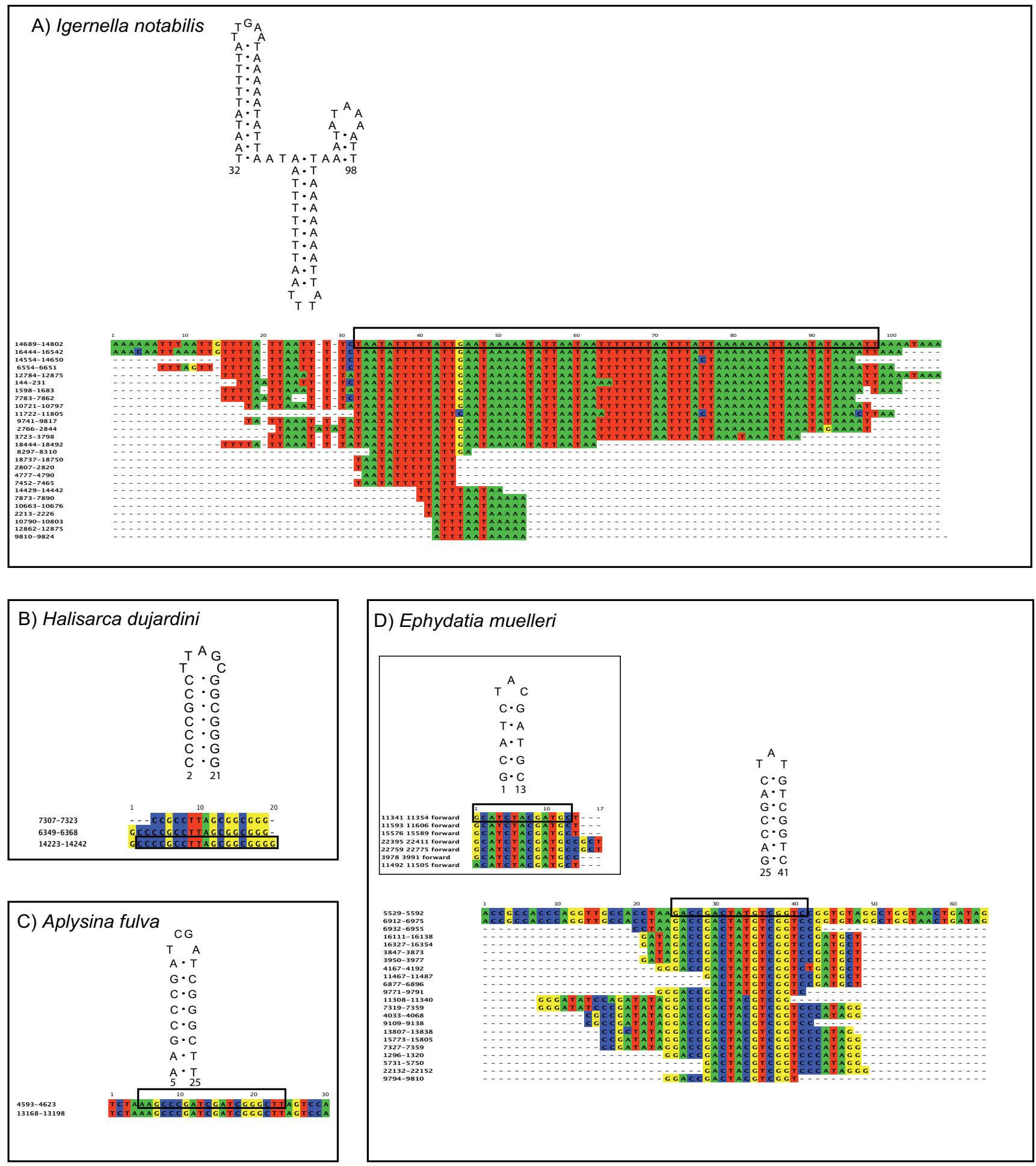

Figure 2

RHEs in miscellaneous demosponges. Secondary structures and their corresponding alignments of RHEs in A) lgernella notabilis (Dendroceratida), B) Halisarca dujardini (Halisarcida), C) Aplysina fulva (Verongida) and D) Ephydatia muelleri (Spongillina). The numbers in the sequence names refer to their position in the $\mathrm{mt}$ genome. The numbers at the structures refer to their position in the alignments. The black box indicates the particular fragment region for which the secondary structure is given. Alignable flanking regions are displayed. 
possesses a large amount of intergenic nucleotides $(5,242$ bp IGR), containing RHEs with equal GC/AT- composition (Figure 2D). The most frequent RHE consists of two 8 bp complementary regions and is present 19 times in IGRs and rnl. Occasionally, RHEs form a triple hairpin structure. The mt genomes of Cinachyrella kuekenthali (490 bp IGR), Geodia neptuni (Astrophorida, 340 bp IGR), Topsentia ophiraphidites (Halichondrida, 1,856 bp IGR), Negombata magnifica (Poecilosclerida, 2,064 bp IGR), Ectyoplasia ferox (Poecilosclerida, 530 bp IGR), Iotrochota birotulata (Poecilosclerida, 1,355 bp IGR), Ptilocaulis walpersi (Halichondrida, 1,003 bp IGR), Agelas schmidti (Agelasida, 2,134 bp IGR) and Tethya actinia (Hadromerida, 1,485 bp IGR) do not possess any peculiar repetitive features according to our search criteria, except for a $91 \mathrm{bp}$ long fragment of nad1 copied into the IGR 5' of nad2 of $I$. birotulata. In contrast, Axinella corrugata (Halichondrida, 6,077 bp IGR) possesses a large variety of GC-rich (>70\%) RHEs, including double and triple hairpin motifs (see Figure 3). In several instances, these repeats are tandemly arranged into multi-repeat motifs. Repeats reach a length up to $84 \mathrm{bp}$ and are present in almost all intergenic regions of A. corrugata, as well as inside nad2, nad5 and both ribosomal RNA genes. For Suberites domuncula (Hadromerida, 6,519 bp IGR), numerous repetitive hairpin-forming features have also been reported earlier [35]. Three different RHE types with balanced nucleotide composition were detected with our search criteria, one of them comprises 30 copies in different fragments sizes of up to 40 bp (Figure 4). The Suberites domuncula RHE are present inside rns, rnl and nad5 and in the IGRs 5' to $\operatorname{trnI}(\mathrm{cau}), \operatorname{trnK}, \operatorname{atp6}, \operatorname{trnR}(\mathrm{ucu}), \operatorname{cox3}, \operatorname{trnQ}(\mathrm{uug})$, $\operatorname{trnN}(g u u), c o b, \operatorname{trnT}(u g u), \operatorname{trnE}(u u c), \operatorname{trn} D(g u c), \operatorname{trnR}(u g c)$, trnH(gug), cox1, trnS(uga), nad1, trnL(uaa), nad2, nad5, $\operatorname{trn} A(u g c), \operatorname{trnF}(g a a)$ and $\operatorname{trnV}(u a c)$.

\section{Repetitive and other unusual elements in mtDNA of other poriferan lineages}

Recent phylogenomic analyses suggested the presence of four major extant sponge lineages: Demospongiae, Calcarea, Hexactinellida and Homoscleromorpha [29].

\section{Hexactinellida}

The mt DNA of Iphiteon panicea and Sympagella nux are not completely sequenced due to the difficulties associated with PCR amplification of a single large non-coding region present in these genomes (see [36]), and hence may contain a number of undetected RHE. Therefore the Hexactinellida results have to be regarded with caution. The sequenced region of Iphiteon panicea (Hexactinosida, 1,551 bp IGR) does not possess any hairpin-forming repeat structures. Instead there are six repetitive regions of about $30 \mathrm{bp}$, which make up in some cases almost an entire IGR and overlap by 5 bp with the 3 ' regions of nad3, nad4L and nad4. A full-length copy of such repeat is inserted within $c o b$. This $3^{\prime}$ region of $c o b$ is highly variable and therefore potentially not relevant for the function of the gene. Other copies of the motif overlap with $\operatorname{trnI}(\mathrm{gau})$ and reside within orf909. Furthermore, several additional repeat units are present in Iphiteon panicea, which share up to $43 \mathrm{bp}$ and a $7 \mathrm{bp}$ core sequence. The complete mtDNA sequence of Aphrocallistes vastus (1,444 bp IGR, including one orf), another representative of the order Hexactinosida, does not possess RHE either. A large non-coding region has been proposed to be a control region [11]. This region contains several repetitive elements including a single (not repetitive) 28 bp hairpin motif consisting of two perfectly complementary 14 bp stretches, one $21 \mathrm{bp}$ complementary repeat and a 21 bp region shared with the IGR upstream of cox2. Other repeated regions include three identical (non RHE-) elements of 41 bp in $r n s, r n l$ and the 5' terminus of cox2. The mt genome of Sympagella nux (Lyssacinosida, 1.011 bp IGR) does not possess RHEs in the sequenced portion, but contains a $117 \mathrm{bp}$ repeated region including trnD, of which a copy is present $423 \mathrm{bp}$ upstream between atp9 and $\operatorname{trnM}(\mathrm{cau})$.

\section{Homoscleromorpha}

The mt genomes of the homoscleromorph Plakortis angulospiculatus (601 bp IGR) and Oscarella carmela (1,275 bp IGR) lack RHE according to our search criteria.

\section{Calcarea}

Yet, we lack any comprehensive information on calcarean (calcareous sponges) mt genomes.

\section{Discussion \\ Major morphological differences are not reflected in molecular distances}

It is remarkable that the mt genomes from three dictyoceratid species possess an identical gene content and gene arrangement and contain several similar IGR regions, because they represent taxa with very different skeletal types. Vaceletia, a taxon with sphinctozoan-like (chambered) bauplan features a massive, "hypercalcified" skeleton. Sphinctozoan-type sponges were metazoans that built large reef structures in the Palaeozoic and Mesozoic, together with other coralline sponges that built massive calcified skeletons with stromatoporoid, chaetetid, and pharetronid grade of construction (e.g. [37]). Vaceletia had been placed as the only extant genus in the order Verticillitida [38] and only molecular data revealed a dictyoceratid relationship of Vaceletia [5,31]. Among extant organisms, Vaceletia is the only animal that possesses this ancient sphinctozoan-type skeleton. Our data reveals that significant morphological difference of the sphinctozoan coralline sponge Vaceletia (with mineral skeleton), compared to the non-sphinctozoan dictyoceratids such as Hippospongia and Ircinia (with an organic skeleton) is not reflected in either nuclear ribosomal RNA genes [31] or in 
Axinella corrugata
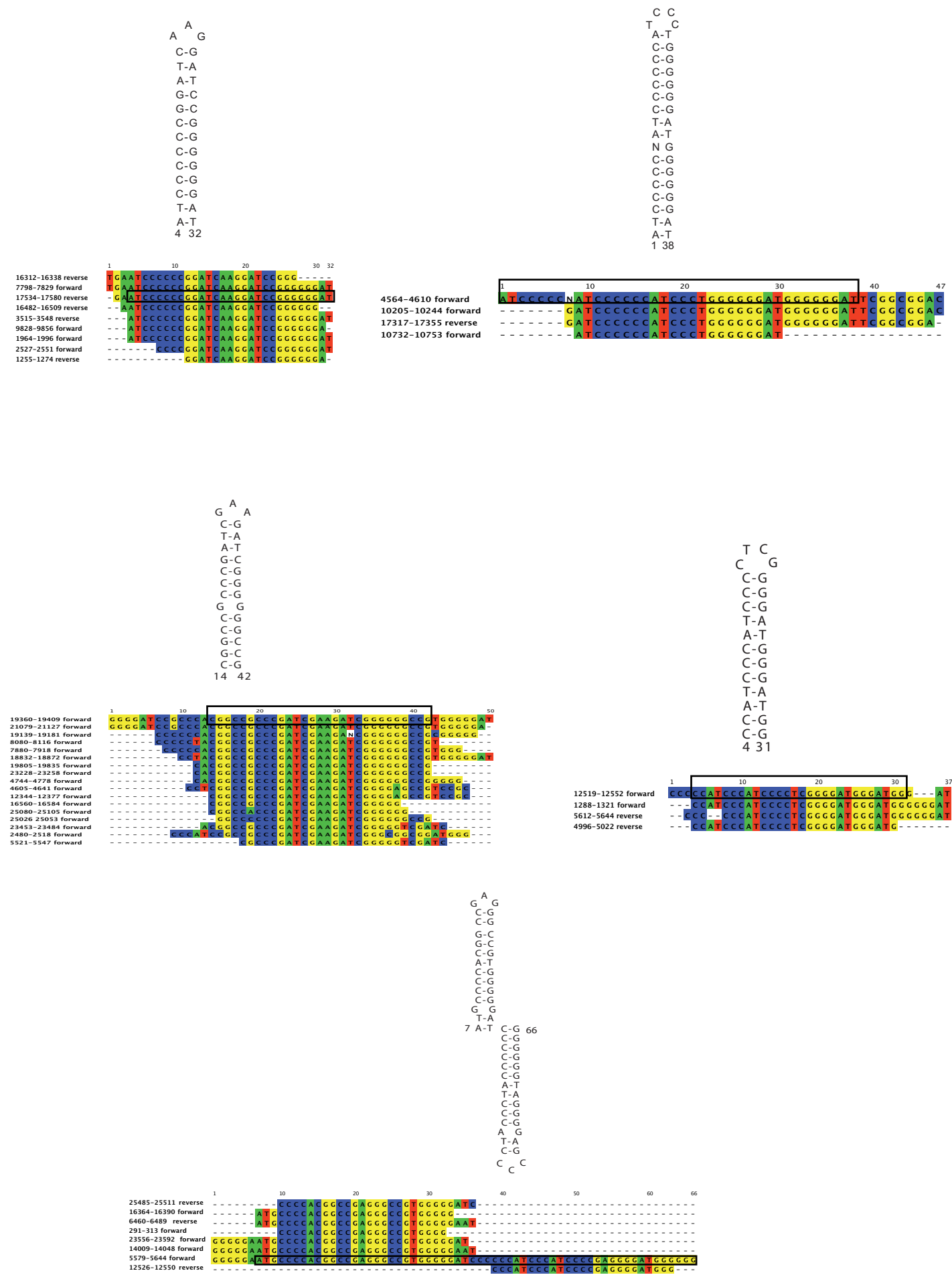

\section{Figure 3}

RHEs in the demosponge Axinella corrugata. Secondary structures and their corresponding alignments of RHEs in Axinella corrugata (Halichondrida). The numbers in the sequence names refer to their position in the mt genome. The numbers at the structures refer to their position in the alignments. The black box indicates the particular fragment region for which the secondary structure is given. Alignable flanking regions are displayed. 


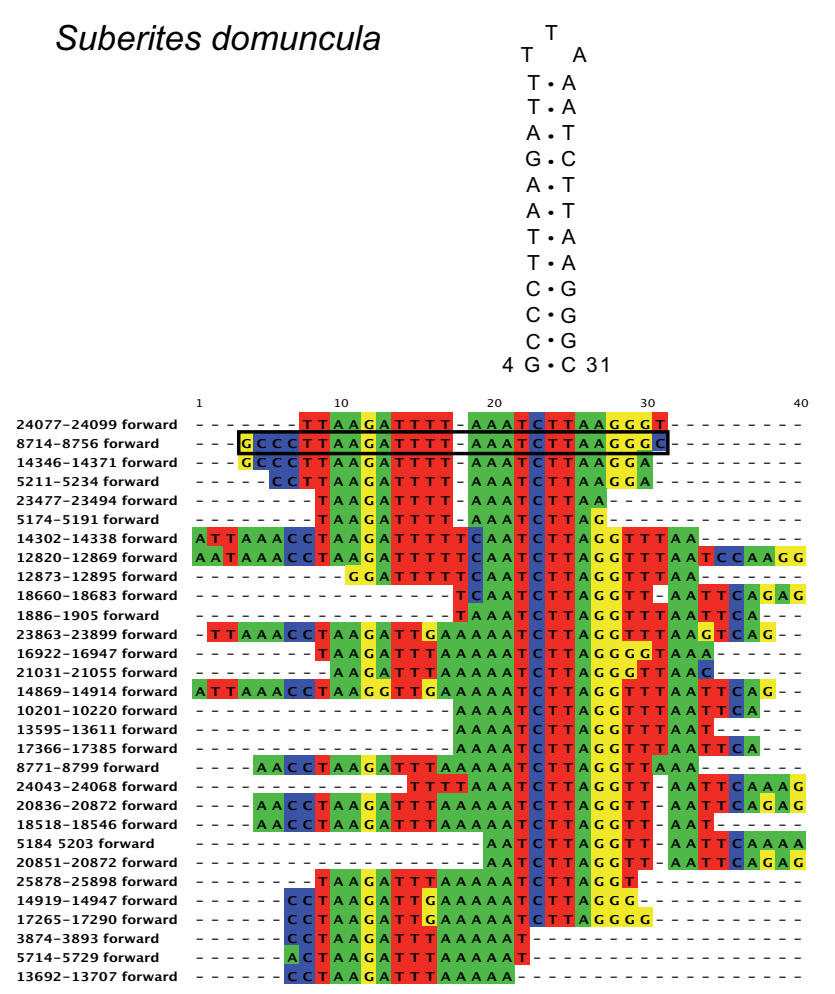

\section{Figure 4}

RHEs in the demosponge Suberites domuncula. Secondary structures and their corresponding alignments of RHEs in Suberites domuncula (Hadromerida). The numbers in the sequence names refer to their position in the $\mathrm{mt}$ genome. The numbers at the structures refer to their position in the alignments. The black box indicates the particular fragment region for which the secondary structure is given. Alignable flanking regions are displayed.

$\mathrm{mt}$ data (Figure 5). This is particularly interesting for understanding molecular phylogenies comprising several other extant coralline demosponges, which likewise construct a 'hypercalcified' secondary limestone skeleton in addition to their primary (often spicular) skeleton [39]. In the past, these coralline sponges had been lumped into a separate sponge class "Sclerospongiae" [40], but were subsequently assigned to several demosponge and calcarean orders, and are clearly polyphyletic [39].

The non-coralline Dictyoceratida Hippospongia and Ircinia share a considerable similarity in IGRs, which may suggest a more recent split between the two taxa. However, these species belong to two different families: Spongiidae and Irciniidae, each with a very distinct morphology: In contrast to Spongiidae, Irciniidae have very fine collagenous filaments with beaded ends in the mesohyl, which supplement the fibre skeleton and are unique among demosponges [41]. Such a well-defined autapomorphy is extremely rare among dictyoceratid sponges [42]. The genetic distances of $\mathrm{mt}$ genes do not indicate a particularly long evolutionary time for Irciniidae to develop this elaborated morphological feature.

The discrepancy between short branch lengths in phylogenetic trees inferred from $\mathrm{mt}$ data and a large extent of morphological divergence can have several explanations. On the one hand, it is possible that the morphological changes have taken place in a relatively short time span. This hypothesis is supported by previous findings on Merlia normani, (Poecilosclerida: Merliidae), a demosponge species known to possesses forms with and without a calcified basal skeleton [43]. The two forms of Merlia normani provided important evidence for understanding that calcified skeletons are frequently homoplasious and therefore weak characters for demosponge phylogenies [43]. Alternatively, the evolutionary rates in Dictyoceratida might have decreased dramatically since their radiation (see Figure 5). Additional keratose sponge $\mathrm{mt}$ data are needed to decide between these two possibilities. 


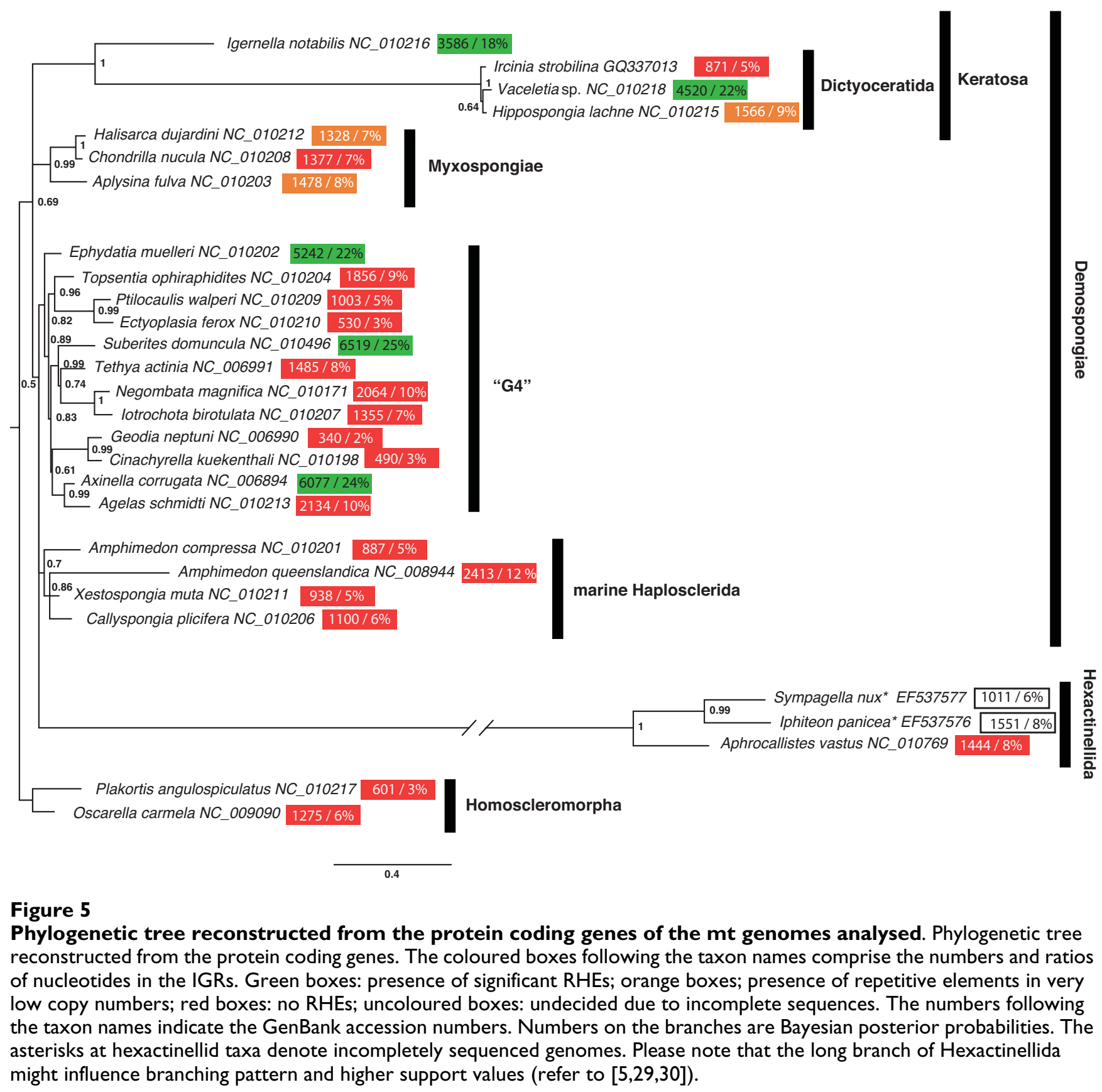

\section{Reduction of the mitochondrial tRNA content in keratose sponges}

The Dictyoceratida and their dendroceratid sister taxon Igernella notabilis retained only two tRNA genes in mtDNA necessary to compensate for the derivation from the universal genetic code: $\operatorname{trn} W(u c a)$ in the sponge (as well as all other animal and many other eukaryote) mitochondria translates the opal codon UGA that specifies a termination signal in the standard genetic code [32]; $\operatorname{trnM}(\mathrm{cau})$ is a specialized initiation of translation (see also [33]). Cnidarian $\mathrm{mt}$ genomes likewise possess an identical set of tRNA genes. However, reduction in the cnidarian and dic- tyoceratid mt tRNA gene sets must have evolved independently given that both Myxospongia, the sister group of Keratosa, and Hexactinellida, the putative sister group of Demospongiae, possess a full complement of mt tRNA genes.

\section{Hairpin elements and the evolution of metazoan mt genomes}

Our study demonstrates that repetitive inverted repeats with potential to form secondary structures such as hairpins, double hairpins or even more elaborate structures, are found repeatedly in demosponge mt genomes. Their 
presence in sponge mt DNA is remarkable because repetitive elements other than in the control region are hardly known from Bilateria (see also [44]). This observation underlines the intermediate appearance of sponge mtDNA $[10,45]$ relative to Eumetazoa, in which such elements are almost unknown, and to non-metazoan opisthokonts such as fungi, in which repetitive elements are abundant.

A correlation between IGR length and the presence of repetitive elements is obvious (Figure 5): RHEs are more common in sponge taxa with an increased IGR length. However, an attempt to explain this correlation leads to the Aristotle's 'Chicken or the Egg?' question: Long IGRs present in most poriferan taxa provide more targets for the accumulation and fixation of RHE, in contrast to the greatly reduced IGRs in Eumetazoa. Insertion of RHE in reduced and highly economized $\mathrm{mt}$ genomes of bilaterian animals could almost exclusively take place in the coding regions only (besides the control region) and would very likely interfere with the functionality of crucial genes. As a consequence, a RHE will less likely be fixed in the population. Vice versa, the accumulation of repetitive elements causes a prolongation of IGRs, which reach up to $25 \%$ of the total genome size in demosponges. Nevertheless, we found many poriferan taxa without significant RHEs in their $\mathrm{mt}$ genomes, but with IGRs of considerable length (e.g. Agelas schmidti, Amphimedon queenslandica, Chondrilla nucula, Negombata magnifica and Iotrochota birotulata), which suggest that RHE are not the sole responsible elements for IGR length.

In a few cases RHEs are present in coding regions of the $\mathrm{mt}$ genomes - mostly in ribosomal RNA genes such as $\mathrm{rnl}$ (Additional file 1) and $r n s$ (Additional file 2), and less frequently in protein genes. Most insertions into ribosomal RNA genes have taken place at sites, in which extensive length differences have been reported earlier, and their presence may not have a significant influence on the function of the ribosomal RNA (see [46]). Therefore, excision of the elements out of the transcript may not be necessary to maintain the function of the RNA in the ribosomes. Studies with fungal $\mathrm{mt}$ double hairpin elements inserted in genes revealed that RHEs are not removed from the transcripts, which is consistent with their absence from structurally important portions of genes [12]. Likewise GC-rich clusters in the var1 ORF or in rRNAs of yeast mitochondria are neither removed nor edited at the RNA level [47-49].

\section{Taxonomic distribution of repetitive hairpin-forming elements in Porifera}

Our analyses leave the question of whether the distribution of RHEs in poriferan $\mathrm{mt}$ genomes has any taxonomic preferences unresolved. It seems possible that some clades are more susceptible to invasions of RHEs than others - but additional poriferan $\mathrm{mt}$ genomes will be necessary to test this hypothesis. So far, Keratosa (c.f. [30]) and, to lesser extend, Myxospongiae (c.f. [30]) display a higher abundance of RHE compared to the clades of marine Haplosclerida and the 'G4' group (c.f. [30]). In the two latter, repetitive hairpin forming elements are only present in Ephydatia muelleri, Axinella corrugata and Suberites domuncula. For Hexactinellida, no unambiguous prediction is possible as only three taxa of two orders were investigated and two out of the three sequences are incomplete. Homoscleromorpha, which is a species-poor taxon [50], do not possess relevant repetitive structural elements, which therefore might not occur in this group. However, one of the two species of homosclermomorphs contains two introns - another type of "selfish" DNA - in cox1 [51].

Repetitive hairpin elements are not uncommon among demosponges and therefore putatively some could have been present in (now extinct) taxa diverging earlier from the lineage leading to the last common ancestor of Porifera. Furthermore, as ancestral mt genomes have likely had larger IGRs, they provided more target sites for insertions of RHEs within a mt genome, it is also possible that RHEs were present in (now extinct) taxa diverging earlier from the lineage towards the last common ancestor of Metazoa. Subsequent genome compression in the lineage towards Bilateria after the split of Porifera combined with the loss of IGRs prevented the infestations of RHE in higher metazoan $\mathrm{mt}$ genomes.

The scattered distribution of RHEs that we observed in the present study could either suggest an early origin with subsequent parallel loss, or multiple independent invasions. The latter possibility appears more plausible given the structural differences between RHE elements found among demosponge taxa. Consequently, repetitive hairpin-forming elements may have invaded metazoan $\mathrm{mt}$ genomes repeatedly during their evolution. They may be secondarily lost again in some poriferan lineages, but are, with the exception of the control region, mostly absent in eumetazoan mt genomes due to their compact organization with subsequent loss of the preferred target sites within the IGRs (but see also [44]).

\section{Evolution of repetitive hairpin-forming elements in Porifera}

Large differences in secondary structures and nucleotide composition observed in RHEs of sponges suggest their independent origin and evolution. RHEs in the keratose sponges Vaceletia, Hippospongia and Igernella have an extremely high AT content. Both "yellow18" and "blue15" motifs in Vaceletia mtDNA likely have a common origin. It is possible that the short fraction of the "yellow18" type RHE evolved into the "blue15" type RHE, of which subsequently several copies independently inserted into other parts of the Vaceletia mt genome. Apparent double hair- 
pins (which are known from other genomes) are likewise formed by tandem insertion of "blue15" and/or "yellow18" RHEs into the $\mathrm{mt}$ genome.

The stem regions of both, "blue15" and "yellow18" RHE types are conserved, while the loop regions display a few differences. We interpret this as an indication of either their recent origin and rapid spread through the genome or considerable pressure for maintaining a hairpin secondary structure and note that this pattern is in contrast with other structured RNAs, such as group I and group II introns $[52,53]$, in which the loops tend to be more conserved than the helical regions [12]. This lack of sequence conservation in the loops in demosponge hairpin elements suggests their lack of structural importance and their unlikely involvement in any tertiary interactions [12]. This observation is supported by the substitution pattern in large repetitive, triple hairpin forming regions of Igernella notabilis, in which helix regions are also conserved and substitutions only occur in the loop regions.

The RHE found in other demosponges have a higher GCcontent. In particular, the stem regions of RHE in Halisarca dujardini are up to $100 \% \mathrm{G}+\mathrm{C}$. Substitutions only occur at the loop positions, which parallels to the structural constraints observed in the keratose RHE.

The lack of similarity between the RHE of different sponge taxa implies that they infested the mt genome in multiple, independent events rather than in a single infestation followed by proliferation into different elements. This inference is supported by the abundance of structural different repetitive hairpin-forming elements in fungi and other non-metazoan opisthokonts. In particular, the distinction into GC-rich and AT-rich elements raises evidence for at least two, but probably more infestation events in Porifera. This is consistent with many earlier observations that well-distinguishable structure forming repetitive elements are frequently confined to groups of closely related species, where the distribution indicates direct exchange of genetic material (see [12] for examples, but also for evidence for mobility).

The relatively conserved structures of sponge RHEs within the individual mt genomes suggest their recent multiplication and dispersal throughout the $\mathrm{mt}$ genome. However, we also have to consider that alternatively, reduced substitution rates in diploblast mt genomes, which are up to 1020 times lower than their bilaterian counterparts [54] may contribute to the low number of base substitutions observed between copies of each element.

\section{Proliferation of repetitive hairpin-forming elements within poriferan mt genomes}

The extensive repetitive and secondary-structure-forming regions in the Vaceletia $\mathrm{mt}$ genome provide insight into the intra-genomic dispersal of the hairpin elements. The large identical IRG clusters indicate that the hairpin elements are not necessarily only copied as single elements. Instead, larger motifs such as the 399 bp repetitive region ("red399 motif", Figure 1) are likewise duplicated and inserted at different positions of the mt genome. Shorter fragments with high sequence identity to those fragments such as the 236 and 219 bp ("red motifs") fragment might be derived from a copy of the 339 bp counterpart and subsequently reduced after insertion as full length elements. The spread of RHEs in Vaceletia was apparently a rapid process compared to (and probably largely independent from) other genomic changes like substitutions and rearrangements in the gene order as evident by comparing the $\mathrm{mt}$ genomes of Vaceletia and Ircinia. The latter genome has an identical gene arrangement, but completely lacks RHEs.

Evidence for lateral transfer and inter-genomic mobility of repetitive hairpin elements could not be found due to the lack of sufficient population samples. In non-metazoan taxa inter-genomic mobility of RHE was hypothesized e.g., for fungi of the genus Allomyces, where closely related species possess different frequencies of RHE insertions [12] in congruence to previous observations in rice [16] and yeast $[13,14]$. Mechanisms for mobility of RHE may be different. Mobility of the yeast RHE, located in GC-rich clusters, is believed to happen by means of transposition at the DNA level similar to DNA transposons [14]. By contrast, a different mode of transposition, potentially via RNA intermediates, has been suggested for the Allomyces RHEs because of the lack of duplications in the flanking regions typical for DNA-transpositions [12]. For Porifera, the lack of mt sequences of closely related species yet prevents speculation on their RHE transposition mechanisms.

\section{Conclusion}

Several poriferan $\mathrm{mt}$ genomes possess large IGRs, which are target sites for repetitive hairpin elements. RHEs themselves also contribute to the large size variation found among sponge mt genomes. Their scattered distribution and dissimilar structure strongly suggests multiple independent invasions of RHEs instead of a single ancestral event with subsequent loss in some lineages. Additionally, the presence of RHE- clusters in Vaceletia sp. implies a rapid proliferation in combination with intra-genomic mobility of such motifs.

As RHEs are not uncommon among extant demosponges, occasional RHE invasions might also have occurred in (now extinct) taxa diverging earlier from the lineage leading to the last common ancestor of Porifera. Furthermore, as ancestral mt genomes were probably richer in IGRs, and therefore provided more target sites for insertion of RHEs, it is likely that occasional RHEs infestations already 
occurred very early in metazoan $\mathrm{mt}$ genome evolution (and affected now extinct lineages). Subsequent genome compression in the lineage towards Bilateria after the split of Porifera combined with the loss of IGRs lead to the loss of RHE in eumetazoan mtDNA.

The mt genomes of Dictyoceratida provide information on metazoan $\mathrm{mt}$ genome evolution. The high nucleotide and structural similarity of the dictyoceratid $\mathrm{mt}$ genomes is opposed to the different morphology of its taxa, which must be accounted for in evolutionary studies on other poriferan groups with a similar degree of morphological differences.

\section{Methods}

Ircinia strobilina was collected by Robert Thacker at the Smithsonian Tropical Research Institute's Bocas del Toro Research Station in Panama. Total DNA of Ircinia strobilina was extracted from $\sim 0.2 \mathrm{~g}$ of tissue with a phenol- chloroform method modified from [55]. Porifera-optimized conserved primers for cox1 and cox2 [5] were used to amplify short fragments of these genes. Two species-specific primers were designed for each gene (is-cox1-f1, 5'GGGAATAAGTTGAACTCGACTGC-3', is-cox1-r1, 5'-TACCGATAGACACCATGGCATAC-3', is-cox2-f1, 5'-AGAGGTGGACAACAGACTATTGC-3', and is-cox2-r1, 5'TGATTTAATCTCCCTGGCACTGC-3') and complete mtDNA was amplified in two fragments 6 and $10.5 \mathrm{kbp}$ in size using the Long and Accurate (LA) PCR kit from TAKARA. The PCR amplifications were combined in equimolar concentration, sheared into pieces $1-2 \mathrm{~kb}$ in size and cloned using the $\mathrm{TOPO}^{\circledR}$ Shotgun Subcloning Kit from Invitrogen. Colonies containing inserts were collected, grown overnight in 96-well blocks and submitted to the DNA Sequencing and Synthesis Facility of the ISU Office of Biotechnology for high-throughput plasmid preparation and sequencing. The STADEN program suite $[56,57]$ was used to basecall and to assemble the sequences. Gaps in the assembly were filled by primerwalking using original PCR amplifications as templates. The repeats observed were too short to interfere negatively with the assembly process; see [58] also for other details of the shotgun plastid sequencing procedure. tRNA genes were identified with the tRNAscan-SE program [59]; other genes were identified by similarity searches in GenBank at NCBI using the BLAST network service [60]. The sequence of Ircinia strobilina is deposited to Genbank under accession number GQ337013.

For the phylogenetic reconstructions protein data of sponge mtDNA was aligned following previously published methods e.g. [5]: amino acid sequences of individual proteins (except atp8) were aligned three times with ClustalW 1.82 [61] using different combinations of opening/extension gap penalties: 10/0.2 (default), 12/4 and 5/
1. The three alignments were compared using SOAP [62], and only positions that were identical among them were included in phylogenetic analyses. The final alignment comprised 3,576 amino acids. The phylogenetic tree of Ircinia strobilina and other complete mt genomes of Porifera (GenBank accession numbers have been incorporated into Figure 5) has been reconstructed with PHYLOBAYES 2.3 under the CAT $+\Gamma$ model [63] with 4 chains and every 100 th tree sampled after a burn-in of 1000 . More than 9000 trees where sampled from each chain and the largest (maxdiff) and mean (meandiff) discrepancy observed across all bipartitions were maxdiff: 0.103701, meandiff: 0.00459985 , which constitutes a good run according to the PHYLOBAYES manual. The rates of synonymous/nonsynonymous codon substitution rates were estimated with PAML 4.1 [64].

Artemis 9.0 [65] was used for genome visualization and handling, Codoncode Aligner v.2.0.6 http://www.codon code.com for alignment. Repetitive features have been screened using PILER v. 1 in combination with PALS v. 1 [66]. In order to minimize false positives, but to perform sufficient thorough analyses we screened for motifs of at least 13 bp lengths with 95\% identity. Positive hits were compared against GenBank with blastN [67] in order to find evidence for functionality or relationship to other published DNA fragments. RHE secondary structures were initially inferred under minimum free energy predictions from the mfold-server http://frontend.bioinfo.rpi.edu/ applications/mfold/cgi-bin/rna-form1.cgi.

\section{List of abbreviations}

bp: basepair(s); IGR: intergenic regions; mt: mitochondrial; RHE: repetitive hairpin-forming element.

\section{Authors' contributions}

DE designed the study, analysed the data and drafted the manuscript. OV participated in the analysis, in particular of the secondary structures and critical revision of the manuscript. GW contributed to the conception and design of the study, and critical revision of the manuscript. DVL determined mtDNA sequence of Ircinia strobilina, provided RHE data for Axinella corrugata, conducted phylogenetic analysis, and contributed to the writing. All authors read and approved the final manuscript.

\section{Additional material}

\section{Additional file 1}

$16 S$ of Axinella corrugata. Secondary structure prediction of the $m t \mathrm{rnl}$ (16S ribosomal RNA gene) of Axinella corrugata as modified from [51]. RHEs are labelled in red. Regions prone to large length differences in $\mathrm{rnl}$ of other eukaryote taxa (cf. [46]) are shaded in grey.

Click here for file

[http://www.biomedcentral.com/content/supplementary/14712164-10-591-S1.EPS] 


\section{Additional file 2}

$12 S$ of Vaceletia sp. Secondary structure prediction of the $m t$ rns (12S ribosomal RNA gene) of Vaceletia sp. RHEs are labelled in red. Regions prone to large length differences in rns of other eukaryote taxa (cf. [46]) are shaded in grey.

Click here for file

[http://www.biomedcentral.com/content/supplementary/14712164-10-591-S2.EPS]

\section{Acknowledgements}

$\mathrm{DE}$ acknowledges financial support of the European Union under a MarieCurie outgoing fellowship (MOIF-CT-2004 Contract No 2882). GW acknowledges funding from the DFG (German Research Foundation), partially through SPPI 174 "Deep Metazoan Phylogeny". This is a contribution from the GeoBio-CenterLMU. DVL acknowledges financial support from lowa State University.

\section{References}

I. Mira A, Ochman H, Moran NA: Deletional bias and the evolution of bacterial genomes. Trends Genet 200I, I7(10):589-596.

2. Lavrov DV: Key transitions in animal evolution: A mitochondrial DNA perspective. Integrative and Comparative Biology 2007, 47(5):734-743.

3. Burger G, Forget L, Zhu Y, Gray MW, Lang BF: Unique mitochondrial genome architecture in unicellular relatives of animals. Proc Natl Acad Sci USA 2003, 100 (3):892-897.

4. Lavrov DV, Lang BF: Transfer RNA gene recruitment in mitochondrial DNA. Trends Genet 2005, 2 I(3): | 29- | 33.

5. Lavrov D, Wang X, Kelly M: Reconstructing ordinal relationships in the Demospongiae using mitochondrial genomic data. Mol Phylogenet Evol 2008, 49(I): I I I-I 24.

6. Ruiz-Pesini E, Lott MT, Procaccio V, Poole JC, Brandon MC, Mishmar $D$, Yi C, Kreuziger J, Baldi P, Wallace DC: An enhanced mitomap with a global mtDNA mutational phylogeny. Nucleic Acids Res 2007, 35:D823-D828.

7. Solignac M, Monnerot M, Mounolou JC: Mitochondrial-DNA evolution in the melanogaster species subgroup of Drospohila. J Mol Evol 1986, 23(I):31-40.

8. Hixson JE, Wong TW, Clayton DA: Both the conserved stemloop and divergent 5 '-flanking sequences are required for initiation at the human mitochondrial origin of light-strand DNA-replication. J Biol Chem 1986, 26 I (5):2384-2390.

9. Van Oppen MJH, Catmull J, McDonald BJ, Hislop NR, Hagerman PJ, Miller DJ: The mitochondrial genome of Acropora tenuis (Cnidaria: Scleractinia) contains a large group I intron and a candidate control region. J Mol Evol 2002, 55(I): I- 13 .

10. Erpenbeck D, Voigt O, Adamski M, Adamska M, Hooper JNA, Wörheide G, Degnan BM: Mitochondrial diversity of early-branching Metazoa is revealed by the complete $\mathrm{mt}$ genome of a haplosclerid demosponge. Mol Biol Evol 2007, 24(I): 19-22.

II. Rosengarten RD, Sperling EA, Moreno MA, Leys SP, Dellaporta SL: The mitochondrial genome of the hexactinellid sponge Aphrocallistes vastu s: Evidence for programmed translational frameshifting. BMC Genomics 2008, 9:33.

12. Paquin B, Laforest MJ, Lang BF: Double-hairpin elements in the mitochondrial DNA of Allomyces: Evidence for mobility. Mol Biol Evol 2000, I7(I I): 1760-1768.

13. Butow RA, Perlman PS, Grossman LI: The unusual varl gene of yeast mitochondrial-DNA. Science 1985, 228(4707): I496-I 501 .

14. Wenzlau JM, Perlman PS: Mobility of two optional G + C-rich clusters of the varl gene of yeast mitochondrial DNA. Genetics 1990, I 26(1):53.

15. Smith DR, Lee RW: The mitochondrial and plastid genomes of Volvox carteri: Bloated molecules rich in repetitive DNA. BMC Genomics 2009, 10:132.

16. Nakazono M, Kanno A, Tsutsumi N, Hirai A: Palindromic repeated sequences (PRSS) in the mitochondrial genome of rice - evidence for their insertion after divergence of the genus Oryza from the other Gramineae. Plant Mol Biol 1994 , 24(2):273-28।.

17. Yin S, Heckman J, Rajbhandary UL: Highly conserved GC rich palindromic DNA-sequences flank transfer RNA genes in Neurospora crassa mitochondria. Cell I98I, 26(3):325-332.

18. Paquin B, Lang BF: The mitochondrial DNA of Allomyces macrogynus: The complete genomic sequence from an ancestral fungus. J Mol Biol 1996, 255(5):688-701.

19. Schuster W, Hiesel R, Isaac PG, Leaver C), Brennicke A: Transcript termini of messenger-RNAs in higher-plant mitochondria. Nucleic Acids Res 1986, I 4( 1 5):5943-5954.

20. Manley JL, Proudfoot NJ: RNA 3' ends - formation and function - meeting review. Genes Dev 1994, 8(3):259-264.

21. Stern DB, Gruissem W: Control of plastid gene-expression - 3' inverted repeats act as messenger-RNA processing and stabilizing elements, but do not terminate transcription. Cell | 987, 5 | (6): I | 45- II 57.

22. Rochaix JD: Post-transcriptional regulation of chloroplast gene expression in Chlamydomonas reinhardtii. Plant Mol Biol 1996, 32(I-2):327-34I.

23. Dombrowski $S$, Brennicke $A$, Binder $S$ : 3 '-inverted repeats in plant mitochondrial mRNAs are processing signals rather than transcription terminators. Embo Journal 1997. 16(16):5069-5076.

24. Dezamaroczy M, Bernardi G: The GC clusters of the mitochondrial genome of yeast and their evolutionary origin. Gene 1986, 4 I(I): I-22.

25. Dieckmann CL, Gandy B: Preferential recombination between GC clusters in yeast mitochondrial-DNA. Embo Journal 1987, 6(I3):4197-4203.

26. Clarkwalker GD: Invivo rearrangement of mitochondrialDNA in Saccharomyces cerevisiae. Proc Natl Acad Sci USA 1989, 86(22):8847-885I.

27. Weiller G, Schueller CME, Schweyen RJ: Putative target sites for mobile $\mathbf{G}+\mathbf{C}$ rich clusters in yeast mitochondrial-DNA - single elements and tandem arrays. Mol Gen Genet 1989, 218(2):272-283.

28. Weiller GF, Bruckner H, Kim SH, Pratje E, Schweyen RJ: A GC cluster repeat is a hotspot for mit- macro-deletions in yeast mitochondrial-DNA. Mol Gen Genet I99|, 226(I-2):233-240.

29. Philippe H, Derelle R, Lopez P, Pick K, Borchiellini C, Boury-Esnault N, Vacelet J, Deniel E, Houliston E, Queinnec E, et al.: Phylogenomics restores traditional views on deep animal relationships. Curr Biol 2009, 19(8):706-7I2.

30. Borchiellini C, Chombard C, Manuel M, Alivon E, Vacelet J, BouryEsnault N: Molecular phylogeny of Demospongiae: Implications for classification and scenarios of character evolution. Mol Phylogenet Evol 2004, 32(3):823-837.

31. Wörheide G: A hypercalcified sponge with soft relatives: Vaceletia is a keratose demosponge. Mol Phylogenet Evol 2008, 47(I):433-438.

32. Watkins RF, Beckenbach AT: Partial sequence of a sponge mitochondrial genome reveals sequence similarity to Cnidaria in cytochrome oxidase subunit II and the large ribosomal RNA subunit. J Mol Evol I999, 48(5):542-554.

33. $\mathrm{Li} Y$, Holmes WB, Appling DR, RajBhandary UL: Initiation of protein synthesis in Saccharomyces cerevisiae mitochondria without formylation of the initiator tRNA. J Bacteriol 2000, 182(10):2886-2892.

34. Beagley CT, Okimoto R, Wolstenholme DR: The mitochondrial genome of the sea anemone Metridium senile (Cnidaria): Introns, a paucity of tRNA genes, and a near-standard genetic code. Genetics 1998, 148(3): 1091-1 108.

35. Lukić-Bilela L, Brandt D, Pojskić N, Wiens M, Gamulin V, Müller WEG: Mitochondrial genome of Suberites domuncula: Palindromes and inverted repeats are abundant in non-coding regions. Gene 2008, 4I 2(I-2): I-II.

36. Haen KM, Lang BF, Pomponi SA, Lavrov DV: Glass sponges and bilaterian animals share derived mitochondrial genomic features: A common ancestry or parallel evolution? Mol Biol Evol 2007, 24(7): 1518-1527.

37. Fagerstrom JA: The palaeobiology of sclerosponges, chaetetids, archaeocyathids and non spicular calcareous sponges. Palaeontogr Am 1984, 54:303-38I.

38. Vacelet J: Recent 'sphinctozoa', order Verticillitida, family Verticillitidae Steinmann, 1882. In Systema porifera a guide to the 
classification of sponges Volume I. Edited by: Hooper JNA, van Soest RWM. New York, Boston, Dordrecht, London, Moscow: Kluwer Academic/Plenum Publishers; 2002: 1097-1098.

39. Reitner J: 'Coralline Spongien' der Versuch einer phylogenetisch-taxonomischen Analyse. PIs I-62. Berliner geowissenschaftliche Abhandlungen Reihe E (Paläobiologie) 1992, I:I-352.

40. Hartman WD, Goreau TF: Jamaican coralline sponges: Their morphology, ecology and fossil relatives. Symp Zool Soc Lond 1970, 25:205-243.

4I. Cook S de C, Bergquist PR: Family Irciniidae Gray, 1867. In Systema porifera a guide to the classification of sponges Volume I. Edited by: Hooper JNA, van Soest RWM. New York, Boston, Dordrecht, London, Moscow: Kluwer Academic/Plenum Publishers; 2002: 1022-I 027.

42. Cook S de C, Bergquist PR: Order Dictyoceratida Minchin, 1900. In Systema porifera a guide to the classification of sponges Volume I. Edited by: Hooper JNA, van Soest RWM . New York, Boston, Dordrecht, London, Moscow: Kluwer Academic/Plenum Publishers; 2002:1021.

43. Van Soest RWM: Deficient Merlia normani Kirkpatrick, from the Curacao reefs. With a discussion on the phylogenetic interpretation of sclerosponges. Bijdragen tot de Dierkunde 1908, 54(2):2II-2I9.

44. lannelli F, Griggio F, Pesole G, Gissi C: The mitochondrial genome of Phallusia mammillata and Phallusia fumigata (Tunicata, Ascidiacea): High genome plasticity at intra-genus level. BMC Evol Biol 2007, 7(I): 155 .

45. Lavrov DV, Forget L, Kelly M, Lang BF: Mitochondrial genomes of two demosponges provide insights into an early stage of animal evolution. Mol Biol Evol 2005, 22(5): | $23|-| 239$.

46. Cannone JJ, Subramanian S, Schnare MN, Collett JR, D'Souza LM, Du YS, Feng B, Lin N, Madabusi LV, Müller KM, et al.: The comparative RNA web (crw) site: An online database of comparative sequence and structure information for ribosomal, intron, and other RNAs. BMC Bioinformatics 2002, 3:2.

47. Sor F, Fukuhara $\mathrm{H}$ : Nature of an inserted sequence in the mitochondrial gene coding for the I5s ribosomal-RNA of yeast. Nucleic Acids Res 1982, 10(5):1625-1633.

48. Sor F, Fukuhara $\mathrm{H}$ : Complete DNA-sequence coding for the large ribosomal-RNA of yeast mitochondria. Nucleic Acids Res 1983, II(2):339-348.

49. Zinn AR, Pohlman JK, Perlman PS, Butow RA: Invivo double-strand breaks occur at recombinogenic $G+C$-rich sequences in the yeast mitochondrial genome. Proc Natl Acad Sci USA 1988, 85(8):2686-2690.

50. Muricy G, Diaz MC: Order Homosclerophorida Dendy, 1905. Family Plakinidae Schulze, 1880. In Systema porifera a guide to the classification of sponges Volume I. Edited by: Hooper JNA, van Soest RWM. New York, Boston, Dordrecht, London, Moscow: Kluwer Academic/Plenum Publishers; 2002:71-82.

51. Wang $X$, Lavrov D: Seventeen new complete mtDNA sequences reveal extensive mitochondrial genome evolution within the Demospongiae. PLoS ONE 2008, 3(7):e2723.

52. Michel $\mathrm{F}$, Umesono $\mathrm{K}$, Ozeki $\mathrm{H}$ : Comparative and functionalanatomy of group-II catalytic introns - a review. Gene 1989, 82(I):5-30.

53. Michel F, Westhof E: Modeling of the 3-dimensional architecture of group-I catalytic introns based on comparative sequence-analysis. J Mol Biol 1990, 216(3):585-610.

54. Shearer TL, Van Oppen MJH, Romano SL, Wörheide G: Slow mitochondrial DNA sequence evolution in the Anthozoa (Cnidaria). Mol Ecol 2002, I I ( I 2):2475-2487.

55. Saghaimaroof MA, Soliman KM, Jorgensen RA, Allard RW: Ribosomal DNA spacer-length polymorphisms in barley - Mendelian inheritance, chromosomal location, and populationdynamics. Proceedings of the National Academy of Sciences of the United States of America-Biological Sciences 1984, 8 I (24):80 I4-80 I8.

56. Ewing B, Green P: Base-calling of automated sequencer traces using phred. II. Error probabilities. Genome Res 1998, 8(3): $186-194$.

57. Staden R: The STADEN sequence analysis package. Mol Biotechnol 1996, 5(3):233-24I.

58. Burger G, Lavrov DV, Forget L, Lang BF: Sequencing complete mitochondrial and plastid genomes. Nature Protocols 2007, 2(3):603-6I4.
59. Lowe TM, Eddy SR: tRNAscan-SE: A program for improved detection of transfer RNA genes in genomic sequence. Nucleic Acids Res 1997, 25(5):955-964.

60. Benson DA, Karsch-Mizrachi I, Lipman DJ, Ostell J, Wheeler DL: Genbank. Nucleic Acids Res 2003, 3 I (I):23-27.

6I. Thompson JD, Higgins DG, Gibson TJ: Clustal W: Improving the sensitivity of progressive multiple sequence alignment through sequence weighting, position-specific gap penalties and weight matrix choice. Nucleic Acids Res 1994, 22(22):4673-4680.

62. Loytynoja A, Milinkovitch MC: SOAP, cleaning multiple alignments from unstable blocks. Bioinformatics 200I, I7(6):573-574.

63. Lartillot N, Philippe $\mathrm{H}$ : A bayesian mixture model for acrosssite heterogeneities in the amino-acid replacement process. Mol Biol Evol 2004, 2 I(6): 1095-I I09.

64. Yang Z: Paml 4: Phylogenetic analysis by maximum likelihood. Mol Biol Evol 2007, 24(8): I586-I59I.

65. Rutherford K, Parkhill J, Crook J, Horsnell T, Rice P, Rajandream M, Barrell B: Artemis: Sequence visualization and annotation. Bioinformatics 16: 944-945. Bioinformatics 2000, 16:944-945.

66. Edgar RC, Myers EW: Piler: Identification and classification of genomic repeats. Bioinformatics 2005, 21 : II52-II58.

67. Altschul SF, Gish W, Miller W, Myers EW, Lipman DJ: Basic local alignment search tool. J Mol Biol 1990, 215(3):403-4I0.
Publish with Bio Med Central and every scientist can read your work free of charge

"BioMed Central will be the most significant development for disseminating the results of biomedical research in our lifetime. "

Sir Paul Nurse, Cancer Research UK

Your research papers will be:

- available free of charge to the entire biomedical community

- peer reviewed and published immediately upon acceptance

- cited in PubMed and archived on PubMed Central

- yours - you keep the copyright

Submit your manuscript here:

http://www.biomedcentral.com/info/publishing_adv.asp
BioMedcentral 